\title{
The Singularity Formation on the Coupled Burgers-Constantin-Lax-Majda System with the Nonlocal Term
}

\author{
Linrui Li ${ }^{1}{ }^{1}$ and Shu Wang ${ }^{2}$ \\ ${ }^{1}$ Basic Courses Department, Institute of Disaster Prevention, Yanjiao, Sanhe, Hebei 065201, China \\ ${ }^{2}$ School of Mathematics and Information Sciences, Guangzhou University, Guangzhou 510006, Guangdong, China \\ Correspondence should be addressed to Linrui Li; linrui020213@163.com
}

Received 9 April 2020; Accepted 12 June 2020; Published 17 July 2020

Academic Editor: Ciprian G. Gal

Copyright (c) 2020 Linrui Li and Shu Wang. This is an open access article distributed under the Creative Commons Attribution License, which permits unrestricted use, distribution, and reproduction in any medium, provided the original work is properly cited.

In this paper, we study the finite-time singularity formation on the coupled Burgers-Constantin-Lax-Majda system with the nonlocal term, which is one nonlinear nonlocal system of combining Burgers equations with Constantin-Lax-Majda equations. We discuss whether the finite-time blow-up singularity mechanism of the system depends upon the domination between the CLM type's vortex-stretching term and the Burgers type's convection term in some sense. We give two kinds of different finite-time blow-up results and prove the local smooth solution of the nonlocal system blows up in finite time for two classes of large initial data.

\section{Introduction}

We study the formation of singularities for the following coupled Burgers-Constantin-Lax-Majda system with the nonlocal term:

$$
\left\{\begin{array}{l}
\partial_{t} u+a u^{p} u_{x}=b u H u, \quad x \in \mathbb{R}, t>0, \\
\left.u\right|_{t=0}=u_{0}
\end{array}\right.
$$

where $a>0, b>0$, and $p>0$ are the given constants and $H$ is the Hilbert transform operator defined by

$$
H f(x)=\frac{1}{\pi} P . V . \int_{-\infty}^{+\infty} \frac{f(y)}{x-y} \mathrm{~d} y,
$$

or defined by the Fourier transform by

$$
\widehat{H u}(\xi)=-i \operatorname{sign}(\xi) \widehat{u}(\xi) .
$$

In the following, we will use the fractional operator $\Lambda^{\beta}$ with $\beta \in(0,1]$, which is defined by $\Lambda^{\beta} \equiv(-\Delta)^{\beta / 2}$ and can be given by

$$
\Lambda^{\beta} u(x)=k_{\beta} \int_{\mathbb{R}} \frac{u(x)-u(y)}{|x-y|^{1+\beta}} \mathrm{d} y,
$$

where $k_{\beta}=\Gamma(1+\beta) \cos ((1-\beta)(\pi / 2)) / \pi$.

System (1) possesses the nonlocal nonlinear term buHu and the local nonlinear term $a u^{p} u_{x}$. If $a=0$, it recovers back to the famous Constantin-Lax-Majda (CLM) system:

$$
\left\{\begin{array}{l}
\omega_{t}=\omega H \omega \\
\omega(x, 0)=\omega_{0}(x)
\end{array}\right.
$$

which is one nonlocal nonlinear system proposed by Constantin et al. in [1] as a simplified 1D system for the 3D vorticity model on the incompressible Euler equation. It should be pointed out that the question on the finite-time singularity formation of the CLM model is considered to be closely related to the most outstanding mathematical open problems on the three-dimensional incompressible Euler equation. In (5), the nonlocal nonlinear term $\omega H \omega$ is one kind of one-dimensional approximation of the vortexstretching term $D(\omega) \omega$ in the three-dimensional incompressible Euler equation. If $b=0$ and $p=1$, system (1) recovers back to the classical Burgers system in the conservation law field, where its general form with the viscosity is given by (see $[2,3])$ 


$$
u_{t}+u u_{x}=v u_{x x}
$$

It is known that the solutions to two systems, both the CLM system and the Burgers system, have the characteristic of finite-time blow-up singularity formation for the smooth solutions with the smooth initial data. When $a>0$ and $b>0$, system (1) possesses both the CLM type's nonlocal term $u H u$ and the (local) generalized Burgers type's convection term $u^{p} u_{x}$.

We also recall some related problems on the finite-time blow-up singularity regimes about some models with the Hilbert transform and the generalized Burgers equations. Gregorio proposed the following system

$$
\left\{\begin{array}{l}
\omega_{t}+v \omega_{x}-v_{x} \omega=0, \\
v_{x}=H \omega,
\end{array}\right.
$$

as another simplified model of the $3 \mathrm{D}$ vorticity version of incompressible inviscid Euler flow in $[4,5]$ and obtained some numeric results which implied that the convection term $v \omega_{x}$ seems to prevent the appearance of finite-time blow-up singularity. Recently, Lei et al. [6] proved that there exist global smooth solutions to system (7) for a class of large nonnegative initial data. Okamoto et al. [7] suggested the generalized CLM system

$$
\left\{\begin{array}{l}
\omega_{t}+a v \omega_{x}-v_{x} \omega=0 \\
v_{x}=H \omega
\end{array}\right.
$$

with $a$ as a parameter. Catro and Córdoba [8] proved the finite-time blow-up singularity for the solution to system (8) when $a<0$ from a class of smooth initial data. Córdoba et al. $[9,10]$ studied the system $\omega_{t}+H \omega \omega_{x}=0$ and obtained the finite-time blow-up results for the smooth initial data. Sakajo [11] studied the CLM equation with the generalized viscosity term $\omega_{t}=\omega H \omega-\nu(-\Delta)^{\alpha / 2} \omega$, where they gave the explicit solution and obtained the finite-time blow-up result for the smooth initial data. Hou et al. [12] investigated the singularity formation of a nonlinear nonlocal system,

$$
\left\{\begin{array}{l}
u_{t}=2 u v, \\
v_{t}=H \omega,
\end{array}\right.
$$

and proved the finite-time blow-up result for a class of large initial data and the global existence result for another initial data for the Cauchy or periodic problem to system (9). Recently, Choi et al. [13] studied the finite-time blowup of a one-dimensional model from the three-dimensional axisymmetric Euler equations and obtained the finite-time blow-up result for the smooth initial data.

Kiselev et al. [14] studied the blow-up and regularity problem for the fractal Burgers equation $\partial_{t} u+u u_{x}=$ $-(-\Delta)^{\beta} u(0 \leq \beta \leq 1)$. Castro et al. [15] studied the singularity formation of a surface wave model $u_{t}+u u_{x}=\Lambda^{\beta} H u$ (where $0 \leq \beta<1$ ) and obtained the finite-time singularity result when $0 \leq \beta<1$. Hur [16] discussed the generalized surface wave model $\partial_{t} u-2 u \partial_{x} u+H^{j} \Lambda^{\gamma} u=0$ (where $j=0$ or $j=1$ and $\gamma \in(0,1))$ and proved the finite-time singularity result.
Hunter and Ifrim [17] discussed the lifespan of the smooth solutions of a Burgers-Hilbert equation $u_{t}+\varepsilon u u_{x}=H u$ over cubically nonlinear time scales on $\epsilon$.

Meanwhile, we remark that there are a lot of research studies carried out on nonlocal different equations. Coclite et al. [18] obtained the formation of singularities in finite time in nonlocal Burgers equations with the time-fractional derivative and employed Burgers equations to model a problem arising in the job market. Wang and Zhang [19] established the nonexistence of positive solutions to nonlocal Lane-Emden equations and obtained the "Fujita index" for nonlocal reaction-diffusion equations, which is new in the blow-up theory. In this paper, motivated by the above results on the CLM system and the generalized Burgers system with the nonlocal operator, we study the finite-time blow-up singularity mechanism of system (1). We would like to discuss whether the finitetime blow-up singularity mechanism of the system depends upon the domination between the CLM type's vortex stretching and the Burgers type's convection in some sense. We will give two kinds of different finite-time blow-up singularity regimes for two classes of initial data. One mechanism seems to be caused by the domination of the vortex-stretching term of the system, see (17)-(19), which is of CLM's finite-time blow-up mechanism type, but another seems to be caused by the domination of the convection term of the system, see (42) and (43), which is of Burgers' finite-time blow-up mechanism type. That is to say that the coupled Burgers-Constantin-Lax-Majda system possesses two kinds of finite-time blow-up singularity regimes of both the Constantin-Lax-Majda system and the Burgers system.

The rest of this paper is as follows. The main results of this paper are given in Section 2, and Section 3 is devoted to the proofs of the main results of Section 2.

\section{Main Results}

In this section, we give the main results of this paper.

Theorem 1. Let $u_{0} \in L^{1}(\mathbb{R}) \cap C_{0}^{1+\delta}(0<\delta<1)$ and $u_{0}(x) \geq \equiv 0(x \in \mathbb{R})$. Also, let $p>0$. Assume that there exists $\alpha_{0} \in \mathbb{R}$ such that

$$
u_{0}\left(\alpha_{0}\right)=0, \quad H u_{0}\left(\alpha_{0}\right)>0 .
$$

Then, there is finite time $T: 0<T<+\infty$ such that the solution $u(x, t)$ to system (1) blows up in finite time.

Theorem 2. Let $u_{0} \in L^{1}(\mathbb{R}) \cap C_{0}^{1+\delta}(0<\delta<1)$ and $u_{0}(x) \geq \equiv 0(x \in \mathbb{R})$. Also, let $p>0$ and $\lambda(1 /(p+1)<\lambda<1)$ be given and $\tilde{u}_{0}>0$ be the solution to the algebra equation

$$
\frac{a b C_{0}}{2} \frac{\tilde{u}_{0}^{p+2}}{\left\|u_{0}\right\|_{L^{1}(\mathbb{R})}}-\frac{b^{2+(2 / p)} C^{2 / p}}{a^{2}}\left\|u_{0}\right\|_{L^{1}(\mathbb{R})}^{2 / p}=0,
$$

where $C_{0}=C_{0}(p, \lambda, \pi)$ and $C=C(p, \lambda, \pi)$ which are given by 


$$
\begin{aligned}
C_{0}(p, \lambda, \pi) & =\frac{[p-(p+1)(1-\lambda)](1-\lambda)}{(p+1) \pi}, \\
C(p, \lambda, \pi) & =\frac{p \cdot 2^{(2 / p)-1}}{(p+2)^{1+(2 / p)}}\left(\frac{(p+1) \pi}{[p-(p+1)(1-\lambda)](1-\lambda)}\right)^{2 / p} .
\end{aligned}
$$

Assume that there exists $\alpha_{0} \in \mathbb{R}$ such that

$$
u_{0}\left(\alpha_{0}\right)>\tilde{u_{0}}, \quad H u_{0}\left(\alpha_{0}\right)>0 \text {. }
$$

Then, there is finite time $T: 0<T<+\infty$ such that the solution $u(x, t)$ to system (1) blows up in finite time.

Remark 1. For the coupled Burgers-CLM equation, it is obvious that the system is $L^{1}$-conservative on the $L^{1}$ norm of the nonnegative solution by using the property of Hilbert transform $\int_{\mathbb{R}} u(x) H u(x) \mathrm{d} x=0$.

Remark 2. Assumption (10) on initial data in Theorem 1 to guarantee the finite-time blow-up mechanism for system (1) is completely the same as the one for CLM system (5). However, assumption (13) on initial data in Theorem 2 is completely different from assumption (10), which yields to another finitetime blow-up mechanism for system (1). That is to say that there are two kinds of the finite-time blow-up regimes. One blow-up regime, given by Theorem 1, is caused by the nonlocal nonlinear term related to the vortex-stretching term, while another regime given by Theorem 2 comes from Burgers' type convection term. This reflects that the modes of the finite-time blow-up regimes may depend upon the domination between the CLM type's vortex-stretching term and the Burgers' type convection term in some sense.

Remark 3. The assumption condition $H u_{0}\left(\alpha_{0}\right)>0$ in (13) can be replaced by $H u_{0}\left(\alpha_{0}\right) \geq 0$, and Theorem 2 still holds.

\section{The Proof of the Main Results}

To finish the proof of the main results, we, firstly, recall some basic properties of the Hilbert transform (see [1]).

\section{Lemma 1}

$$
\begin{aligned}
H(H f) & =-f, \\
H(f H g+g H f) & =(H f)(H g)-f g, \\
(H f)_{x} & =H\left(f_{x}\right), \\
\int_{\mathbb{R}} u H u(x) \mathrm{d} x & =0 .
\end{aligned}
$$

Now, we give the proof of Theorem 1 by using Lemma 1.

Proof of Theorem 1. For the local existence and uniqueness of the smooth solution in the class of $C\left([0, T], C_{0}^{1+\delta}(\mathbb{R})\right)$ to system (1), it can be obtained by the iteration technique and by using the existence and uniqueness theory of the linearized hyperbolic equation. This is standard, and we omit it here. Also, it is obvious that the solution $u(x, t) \geq 0$ only if $u_{0}(x) \geq 0$.

Taking the Hilbert operator on (1), we get, with the help of Lemma 1, that

$$
H u_{t}+a H\left(u^{p} u_{x}\right)=\frac{1}{2} b\left[(H u)^{2}-u^{2}\right]
$$

Taking $x=\alpha_{0}$ in (16) and using assumption (10) on the initial data, we get

$$
\frac{\mathrm{d}(H u)\left(\alpha_{0}, t\right)}{\mathrm{d} t}=\frac{1}{2} b(H u)^{2}\left(\alpha_{0}, t\right)-a H\left(u^{p} u_{x}\right)\left(\alpha_{0}, t\right),
$$

$$
\begin{aligned}
-a H\left(u^{p} u_{x}\right)\left(\alpha_{0}, t\right) & =-\frac{a}{(p+1) \pi} \int_{\mathbb{R}} \frac{u^{p+1}\left(\alpha_{0}, t\right)-u^{p+1}(y, t)}{|x-y|^{2}} \mathrm{~d} y \\
& =\frac{a}{(p+1) \pi} \int_{\mathbb{R}} \frac{u^{p+1}(y, t)}{|x-y|^{2}} \mathrm{~d} y \geq 0 .
\end{aligned}
$$

Combining (17) and (18), we have

$$
\frac{\mathrm{d}(H u)\left(\alpha_{0}, t\right)}{\mathrm{d} t} \geq \frac{b}{2}\left((H u)\left(\alpha_{0}, t\right)\right)^{2} .
$$

According to the theory on the nonlinear ODE and by using $H u_{0}\left(\alpha_{0}\right)>0$ in assumption (10) on the initial data, we can get from (19) that $H u\left(\alpha_{0}, t\right)$ blows up in finite time.

This completes the proof of Theorem 1 .

Next, to prove Theorem 2, we give a local nonnegative lower bound estimate on the nonlocal term $u^{p}(x) \Lambda u(x)-$ $(1 / p+1) \Lambda u^{1 / p+1}(x)$, which plays the key role on proving the main result of Theorem 2 of this paper. In fact, it is an extension version of Lemma 2 in the paper [15], where the case that $p=1$ is obtained. For completeness, we give its proof here.

Lemma 2. Let $u \in L^{1}(\mathbb{R})$ with $u \geq 0$, $\equiv 0$, and let $p>0$ and $\lambda((1 / p+1)<\lambda<1)$ be given. Then, for any fixed point $x \in \mathbb{R}$ satisfying $u(x)>0$, we have

$$
u^{p}(x) \Lambda u(x)-\frac{1}{p+1} \Lambda u^{p+1}(x) \geq C_{0} \frac{u^{p+2}(x)}{\|u\|_{L^{1}(R)}},
$$

where $C_{0}$ is given by (12) in Theorem 2.

Proof. Let $\Omega$ be the set

$$
\Omega=\{y \in R|| y-x \mid<\Delta\}
$$

where $\Delta>0$ will be given in the following. And let $\Omega_{1}$ and $\Omega_{2}$ be the subsets of $\Omega$ such that

$$
\begin{aligned}
\Omega_{1} & =\{y \in \Omega \mid u(x)-u(y) \geq \lambda u(x)\} \\
& =\{y \in \Omega \mid u(y) \leq(1-\lambda) u(x)\}, \\
\Omega_{2} & =\{y \in \Omega \mid u(x)-u(y)<\lambda u(x)\} \\
& =\{y \in \Omega \mid u(y)>(1-\lambda) u(x)\} .
\end{aligned}
$$

In the following, $\Delta>0$ will be chosen to be such that $\left|\Omega_{1}\right|>0$ and $\left|\Omega_{2}\right|>0$. Obviously, $0<\left|\Omega_{1}\right|=|\Omega|-\left|\Omega_{2}\right| \leq$ $|\Omega|=2 \Delta$. 
On the one hand, using the fact

we have

$$
\begin{aligned}
\Lambda u(x)=\frac{1}{\pi} \int_{\mathbb{R}} \frac{u(x)-u(y)}{|x-y|^{2}} \mathrm{~d} y & , \\
u^{p}(x) \Lambda u(x)-\frac{1}{p+1} \Lambda u^{p+1}(x) & =\frac{1}{\pi} \int_{\mathbb{R}} \frac{u^{p}(x)(u(x)-u(y))}{|x-y|^{2}} d y-\frac{1}{p+1} \frac{1}{\pi} \int_{\mathbb{R}} \frac{u^{p+1}(x)-u^{p+1}(y)}{|x-y|^{2}} \mathrm{~d} y \\
& =\frac{1}{p+1} \frac{1}{\pi} \int_{\mathbb{R}} \frac{p u^{p+1}(x)-(p+1) u^{p}(x) u(y)+u^{p+1}(y)}{|x-y|^{2}} \mathrm{~d} y .
\end{aligned}
$$

By Young's inequality $a b \leq a^{p}+\left(b^{q} / q \cdot p^{q / p}\right)$ with $(1 / p)+(1 / q)=1$, we get

$$
(p+1) u^{p}(x) u(y) \leq u^{p+1}(y)+p u^{p+1}(x),
$$

which yields to

$$
\frac{p u^{p+1}(x)-(p+1) u^{p}(x) u(y)+u^{p+1}(y)}{|x-y|^{2}} \geq 0 .
$$

Combining (24) with (26), we have

$$
\begin{aligned}
& u^{p}(x) \Lambda u(x)-\frac{1}{p+1} \Lambda\left(u^{p+1}(x)\right) \\
& \geq \frac{1}{p+1} \frac{1}{\pi} \int_{\Omega_{1}} \frac{p u^{p+1}(x)-(p+1) u^{p}(x)(1-\lambda) u(x)}{\Delta^{2}} \mathrm{~d} y \\
& \quad=\frac{[p-(p+1)(1-\lambda)]}{(p+1) \pi \Delta^{2}} u^{p+1}(x)\left|\Omega_{1}\right| .
\end{aligned}
$$

Here, we need $p-(p+1)(1-\lambda)>0$, i.e., $1 /(p+$ 1) $<\lambda<1$.

On the other hand, we have

$$
\begin{aligned}
\|u\|_{L^{1}(\mathbb{R})}= & \int_{\mathbb{R}}|u(y)| \mathrm{d} y=\int_{\mathbb{R}} u(y) \mathrm{d} y \\
& \geq \int_{\Omega_{2}} u(y) \mathrm{d} y \geq u(x)(1-\lambda)\left|\Omega_{2}\right|,
\end{aligned}
$$

which yields to

$$
\left|\Omega_{2}\right| \leq \frac{\|u\|_{L^{1}(R)}}{u(x)(1-\lambda)} .
$$

Since $\left|\Omega_{1}\right|=|\Omega|-\left|\Omega_{2}\right|$ and $|\Omega|=2 \Delta$, we have, by combining (27) and (29), that

$$
\begin{aligned}
& u^{p}(x) \Lambda u(x)-\frac{1}{p+1} \Lambda\left(u^{p+1}(x)\right) \\
& \quad \geq \frac{[p-(p+1)(1-\lambda)]}{(p+1) \pi \Delta^{2}} u^{p+1}(x)\left|\Omega_{1}\right| \\
& =\frac{[p-(p+1)(1-\lambda)]}{(p+1) \pi \Delta^{2}} u^{p+1}(x)\left(|\Omega|-\left|\Omega_{2}\right|\right) \geq \frac{[p-(p+1)(1-\lambda)]}{(p+1) \pi \Delta^{2}} u^{p+1}(x)\left(|\Omega|-\frac{\|u(x)\|_{L^{1}(R)}}{u(x)(1-\lambda)}\right) \\
& =\frac{[p-(p+1)(1-\lambda)]}{(p+1) \pi \Delta^{2}} u^{p+1}(x)\left(2 \Delta-\frac{\|u(x)\|_{L^{1}(R)}}{u(x)(1-\lambda)}\right) .
\end{aligned}
$$

Now, we choose $\Delta=\|u\|_{L^{1}(\mathbb{R})} /(1-\lambda) u(x)$ for fixed $x \in \mathbb{R}$ satisfying $u(x)>0$. Thus, we get from (30) that

$$
\begin{aligned}
& u^{p}(x) \Lambda u(x)-\frac{1}{p+1} \Lambda\left(u^{p+1}(x)\right) \\
& \geq \frac{[p-(p+1)(1-\lambda)]}{(p+1) \pi \Delta^{2}} u^{p+1}(x)\left(2 \Delta-\frac{\|u(x)\|_{L^{1}(R)}}{u(x)(1-\lambda)}\right) \\
& \quad=\frac{[p-(p+1)(1-\lambda)](1-\lambda)}{(p+1) \pi\|u\|_{L^{1}(\mathbb{R})}} u^{p+2}(x),
\end{aligned}
$$

which gives (20). This completes the proof of Lemma 2.

Remark 4. Under the assumption of Lemma 2, inequality (20) holds still for the point $x_{0} \in \mathbb{R}$ such that $u\left(x_{0}\right)=0$ because $u^{p}\left(x_{0}\right) \Lambda u\left(x_{0}\right)-(1 /(p+1)) \Lambda u^{p+1}\left(x_{0}\right)=-1 /(p+$ 1) $\Lambda u^{p+1}\left(x_{0}\right)=1 /(p+1) \int_{\mathbb{R}}\left(u^{p+1}(y) /\left|x_{0}-y\right|^{2}\right) \mathrm{d} y \geq 0$.

Proof of Theorem 2. First, it is easy to prove that there is a unique smooth solution $u(x, t) \in C\left([0, T], C_{0}^{1+\delta}(\mathbb{R})\right)$ of system (1) for some time $0<T \leq \infty$.

Now, we want to prove the solution will blow up in finite time for some classes of large initial data in the sense of our 
assumption. We end this by a contradiction argument. Assume that there exists the global solution for all time $t \in(0, \infty)$.

Firstly, for the solution of system (1), there exists one $L^{1}$ conservation law, see Remark 1:

$$
\|u(t)\|_{L^{1}(\mathbb{R})}=\left\|u_{0}\right\|_{L^{1}(\mathbb{R})} .
$$

Then, we define the particle trajectories $x(\alpha, t)$ by the equation

$$
\left\{\begin{array}{l}
\frac{\mathrm{d} x(\alpha, t)}{\mathrm{d} t}=a u^{p}(x(\alpha, t), t), \\
\left.x(\alpha, t)\right|_{t=0}=\alpha .
\end{array}\right.
$$

It follows from systems (1) and (33) that

$$
\frac{\mathrm{d}(u(x(\alpha, t), t))}{\mathrm{d} t}=b(u H u)(x(\alpha, t), t),
$$

which yields to

$$
u(x(\alpha, t), t)=u_{0}(\alpha) e^{b \int_{0}^{t}(H u)(x(\alpha, s), s) \mathrm{d} s} .
$$

Thus, it follows from (35) that $u(x(\alpha, t), t) \geq 0$ for any $t \in(0, \infty)$ if $u_{0}(\alpha) \geq 0$ for some fixed $\alpha \in \mathbb{R}$, and $u(x(\alpha, t), t)>0$ for any finite $t \in(0, \infty)$ if $u_{0}(\alpha)>0$ for some fixed $\alpha \in \mathbb{R}$.

For any fixed $\alpha \in \mathbb{R}$ satisfying $u_{0}(\alpha)>0$ (especially, this is true for $\alpha=\alpha_{0}$ in Theorem 2), it follows from (34) that

$$
\frac{\mathrm{d} \log u(x(\alpha, t), t)}{\mathrm{d} t}=b(H u)(x(\alpha, t), t),
$$

and, hence, by using (16), (32), and (20) in Lemma 2, we have

$$
\begin{aligned}
\frac{\mathrm{d}^{2} \log u(x(\alpha, t))}{\mathrm{d} t^{2}}= & b\left[\partial_{t}(H u)(x(\alpha, t), t)+\partial_{x}(H u)(x(\alpha, t), t) a u^{p}(x(\alpha, t), t)\right] \\
= & b\left[b H(u H u)-H\left(a u^{p} u_{x}\right)\right](x(\alpha, t), t)+a b u^{p} \Lambda u(x(\alpha, t), t) \\
= & \left(b^{2} H(u H u)-a b H\left(\frac{u^{p+1}}{p+1}\right)_{x}+a b u^{p} \Lambda u\right)(x(\alpha, t), t) \\
= & b^{2} H(u H u)(x(\alpha, t), t)+a b\left(u^{p} \Lambda u-\frac{1}{p+1} \Lambda u^{p+1}\right)(x(\alpha, t), t) \\
& \geq b^{2} H(u H u)(x(\alpha, t), t)+a b C_{0} \frac{u^{p+2}(x((\alpha, t), t)}{\left\|u_{0}\right\|_{L^{1}(R)}} \\
= & \frac{b^{2}}{2}\left((H u)^{2}-u^{2}\right)(x(\alpha, t), t)+a b C_{0} \frac{u^{p+2}(x(\alpha, t), t)}{\left\|u_{0}\right\|_{L^{1}(R)}} .
\end{aligned}
$$

Here, we have used $H\left(u^{p} u_{x}\right)(x)=1 /(p+$ 1) $H\left(\left(u^{p+1}\right)_{x}\right)=(1 /(p+1)) \Lambda\left(u^{p+1}\right)(x), \quad \Lambda u=H u_{x}$, and $H(u H u)=(1 / 2)\left[(H u)^{2}-u^{2}\right]$.

By Young's inequality, we have

$$
\frac{b^{2}}{2} u^{2} \leq \frac{a b C_{0}}{2} \frac{u^{p+2}}{\left\|u_{0}\right\|_{L^{1}(\mathbb{R})}}+\frac{b^{2+(2 / p)}}{a^{2 / p}} C(p, \lambda, \pi)\left\|u_{0}\right\|_{L^{1}(\mathbb{R})}^{2 / p},
$$

where $C(p, \lambda, \pi)>0$ is given by (13) in Theorem 2 .

Putting (38) into (37), we have

$$
\begin{aligned}
\frac{\mathrm{d}^{2} \log u(x(\alpha, t), t)}{\mathrm{d} t^{2}} \geq & \frac{a b C_{0}}{2} \frac{u^{p+2}(x(\alpha, t), t)}{\left\|u_{0}\right\|_{L^{1}(\mathbb{R})}} \\
& -\frac{b^{2+(2 / p)}}{a^{2 / p}} C(p, \lambda, \pi)\left\|u_{0}\right\|_{L^{1}(\mathbb{R})}^{2 / p}
\end{aligned}
$$

$$
\frac{\mathrm{d} \log u(x(\alpha, t), t)}{\mathrm{d} t}=V(x(\alpha, t), t),
$$
then, by (39) and (40), we have

$$
\left\{\begin{array}{l}
\frac{d u(x(\alpha, t), t)}{d t}=u(x(\alpha, t), t) V(x(\alpha, t), t), \\
\frac{\mathrm{d} V(x(\alpha, t), t)}{\mathrm{d} t} \geq \frac{a b C_{0}}{2} \frac{u^{p+2}(x(\alpha, t), t)}{\left\|u_{0}\right\|_{L^{1}(\mathbb{R})}} \\
-\frac{b^{2+(2 / p)}}{a^{2 / p}} C(p, \lambda, \pi)\left\|u_{0}\right\|_{L^{1}(\mathbb{R})}^{2 / p} \\
\left.(u(x(\alpha, t), t), V(x(\alpha, t), t))\right|_{t=0}=\left(u_{0}(\alpha), V_{0}(\alpha)\right) .
\end{array}\right.
$$

Now, setting 
By the existence-uniqueness and extension theory of ordinary differential equations, we know that the solution $(u, V)(x(\alpha, t), t)$ of system $(40)$ is nondecreasing with respect to the time $t \geq 0$ and for fixed $\alpha$ and satisfies $(u, V)(x(\alpha, t), t) \geq\left(\tilde{u}_{\theta} 0\right) \quad$ and $\quad(u, V)(x(\alpha, t), t) \longrightarrow$ $(+\infty,+\infty)$ as $t \longrightarrow+\infty$ if $\left(u_{0}, V_{0}\right)(\alpha)>\left(\tilde{u}_{\theta} 0\right)$ for some fixed $\alpha \in \mathbb{R}$, where $u=\tilde{u}_{0}$ is the solution to algebra equation (11). In fact, here, $V_{0}(\alpha)=b H u_{0}(\alpha)$ by using (36).
Taking $\alpha=\alpha_{0}$ especially, where $\alpha_{0}$ is given in Theorem 2, by the assumption on Theorem 2 , we have that $\left(u_{0}, V_{0}\right)$ $\left(\alpha_{0}\right)>\left(\tilde{u}_{0}, 0\right)$. Hence, it is obvious that there exists time $t_{0}>0$ such that $u\left(x\left(\alpha_{0}, t\right), t\right)$ is nondecreasing for $t \geq t_{0}>0$, and $(u, V)\left(x\left(\alpha_{0}, t_{0}\right), t_{0}\right)$ satisfies $\left(a b C_{0} / 4\right)\left(u^{p+2}\left(x\left(\alpha_{0}, t_{0}\right), t_{0}\right) /\right.$ $\left.\left\|u_{0}\right\|_{L^{1}(\mathbb{R})}\right) \geq\left(b^{2+(2 / p)} / a^{2 / p}\right) C(p, \lambda, \pi)\left\|u_{0}\right\|_{L^{1}(\mathbb{R})}^{2 / p}, \quad V\left(x\left(\alpha_{0}, t_{0}\right)\right.$, $\left.t_{0}\right)=b H u\left(x\left(\alpha_{0}, t_{0}\right), t_{0}\right)>0$, and $\log u\left(x\left(\alpha_{0}, t_{0}\right), t_{0}\right)>0$.

Also, taking $\alpha=\alpha_{0}$ in (39), we have, for all $t \geq t_{0}>0$, that

$$
\begin{aligned}
& \frac{\mathrm{d}^{2} \log u\left(x\left(\alpha_{0}, t\right), t\right)}{\mathrm{d} t^{2}} \\
& \quad \geq \frac{a b}{2} \frac{C_{0}}{\left\|u_{0}\right\|_{L^{1}(\mathbb{R})}} u^{p+2}\left(x\left(\alpha_{0}, t\right), t\right)-\frac{b^{2+(2 / p)}}{a^{2 / p}} C(p, \lambda, \pi)\left\|u_{0}\right\|_{L^{1}(\mathbb{R})}^{2 / p} \\
& =\frac{a b}{4} \frac{C_{0}}{\left\|u_{0}\right\|_{L^{1}(\mathbb{R})}} u^{p+2}\left(x\left(\alpha_{0}, t\right), t\right)+\frac{a b}{4} \frac{C_{0}}{\left\|u_{0}\right\|_{L^{1}(\mathbb{R})}} u^{p+2}\left(x\left(\alpha_{0}, t\right), t\right) \\
& -\frac{b^{2+(2 / p)}}{a^{2 / p}} C(p, \lambda, \pi)\left\|_{0}\right\|_{L^{1}(\mathbb{R})}^{2 / p} \\
& \geq \frac{a b}{4} \frac{C_{0}}{\left\|u_{0}\right\|_{L^{1}}(\mathbb{R})} u^{p+2}\left(x\left(\alpha_{0}, t\right), t\right)+\frac{a b}{4} \frac{C_{0}}{\left\|u_{0}\right\|_{L^{1}(\mathbb{R})}} u^{p+2}\left(x\left(\alpha_{0}, t_{0}\right), t_{0}\right) \\
& \quad-\frac{b^{2+(2 / p)}}{a^{2 / p}} C(p, \lambda, \pi)\left\|u_{0}\right\|_{L^{1}(\mathbb{R})}^{2 / p} \geq \frac{a b}{4} \frac{C_{0}}{\left\|u_{0}\right\|_{L^{1}(\mathbb{R})}} u^{p+2}\left(x\left(\alpha_{0}, t\right), t\right) .
\end{aligned}
$$

Integrating (42) with respect to $t$ from $t_{0}$ to $t$, we have

$$
\begin{aligned}
\frac{\mathrm{d} \log u\left(x\left(\alpha_{0}, t\right), t\right)}{\mathrm{d} t} \geq & V\left(x\left(\alpha_{0}, t_{0}\right), t_{0}\right)+\frac{a b C_{0}}{4\left\|u_{0}\right\|_{L^{1}(\mathbb{R})}} \\
& \cdot \int_{t_{0}}^{t} u^{p+2}\left(x\left(\alpha_{0}, \tau\right), \tau\right) \mathrm{d} \tau,
\end{aligned}
$$

which, by integrating (43) with respect to $t$ from $t_{0}$ to $t$, gives

$$
\begin{aligned}
& \log u\left(x\left(\alpha_{0}, t\right), t\right) \geq \log u\left(x\left(\alpha_{0}, t_{0}\right), t_{0}\right) \\
& +V\left(x\left(\alpha_{0}, t_{0}\right), t_{0}\right)\left(t-t_{0}\right) \\
& +\frac{a b C_{0}}{4\left\|u_{0}\right\|_{L^{1}(\mathbb{R})}} \int_{t_{0}}^{t} \int_{t_{0}}^{s} u^{p+2}\left(\left(x\left(\left(\alpha_{0}, \tau\right)\right), \tau\right)\right) \mathrm{d} \tau \mathrm{d} s .
\end{aligned}
$$

Since $u(x(\alpha, t), t) \geq 0$, we have $\log u(x(\alpha, t), t) \leq \log ^{+} u(x(\alpha, t), t) \leq u(x(\alpha, t), t)$.

Combining (44) with (45), we have $u\left(x\left(\alpha_{0}, t\right), t\right) \geq \log u\left(x\left(\alpha_{0}, t\right), t\right) \geq V\left(x\left(\alpha_{0}, t_{0}\right), t_{0}\right)\left(t-t_{0}\right)$

$$
+\frac{a b C_{0}}{4\left\|u_{0}\right\|_{L^{1}(\mathbb{R})}} \int_{t_{0}}^{t} \int_{t_{0}}^{s} u^{p+2}\left(x\left(\alpha_{0}, \tau\right), \tau\right) \mathrm{d} \tau \mathrm{d} s .
$$

Let

$$
\begin{aligned}
F(t)= & V\left(x\left(\alpha_{0}, t_{0}\right), t_{0}\right)\left(t-t_{0}\right) \\
& +\frac{a b C_{0}}{4\left\|u_{0}\right\|_{L^{1}(\mathbb{R})}} \int_{t_{0}}^{t} \int_{t_{0}}^{s} u^{p+2}\left(x\left(\alpha_{0}, \tau\right), \tau\right) \mathrm{d} \tau \mathrm{d} s .
\end{aligned}
$$

Then, by (46), we have

$$
\left\{\begin{array}{l}
F^{\prime \prime}(t) \geq \frac{a b C_{0}}{4\left\|u_{0}\right\|_{L^{1}(\mathbb{R})}} F^{p+2}(t), \quad t \geq t_{0}, \\
F\left(t_{0}\right)=0,\left.F^{\prime}(t)\right|_{t=t_{0}}=V\left(x\left(\alpha_{0}, t_{0}\right), t_{0}\right) .
\end{array}\right.
$$

Multiplying the first equation of (47) by $F^{\prime}(t) \geq 0$ and then integrating the resulting equation, we have 


$$
\begin{aligned}
F^{\prime}(t) & \geq \sqrt{\frac{a b C_{0}}{2(p+3)\left\|u_{0}\right\|_{L^{1}(\mathbb{R})}} F^{p+3}(t)+V^{2}\left(x\left(\alpha_{0}, t_{0}\right), t_{0}\right)} \\
& =V\left(x\left(\alpha_{0}, t_{0}\right), t_{0}\right) \sqrt{\frac{a b C_{0}}{2(p+3)\left\|u_{0}\right\|_{L^{1}(\mathbb{R})} V^{2}\left(x\left(\alpha_{0}, t_{0}\right), t_{0}\right)} F^{p+3}(t)+1} \\
& =V\left(x\left(\alpha_{0}, t_{0}\right), t_{0}\right) \sqrt{\left(\left(\frac{a b C_{0}}{2(p+3)\left\|u_{0}\right\|_{L^{1}(\mathbb{R})} V^{2}\left(x\left(\alpha_{0}, t_{0}\right), t_{0}\right)}\right)^{1 /(p+3)} F(t)\right)^{p+3}+1 .}
\end{aligned}
$$

Let $G=\left(a b C_{0} /\left(2(p+3)\left\|u_{0}\right\|_{L^{1}(\mathbb{R})} V^{2}\left(x\left(\alpha_{0}, t_{0}\right), t_{0}\right)\right)\right)^{1 /(p+3)}$

$F(t)$; we have

$$
G^{\prime}(t) \geq\left(\frac{a b C_{0}}{2(p+3)\left\|u_{0}\right\|_{L^{1}(\mathbb{R})} V^{2}\left(x\left(\alpha_{0}, t_{0}\right), t_{0}\right)}\right)^{1 /(p+3)} V\left(x\left(\alpha_{0}, t_{0}\right), t_{0}\right) \sqrt{G^{p+3}(t)+1} .
$$

That is to say

$$
\frac{G^{\prime}(t)}{\sqrt{G^{p+3}(t)+1}} \geq\left(\frac{a b C_{0}}{2(p+3)\left\|u_{0}\right\|_{L^{1}(\mathbb{R})} V^{2}\left(x\left(\alpha_{0}, t_{0}\right), t_{0}\right)}\right)^{1 /(p+3)},
$$

and, hence,

$$
\left(\int_{t_{0}}^{G(t)} \frac{d \xi}{\sqrt{\xi^{p+3}+1}}\right)^{\prime} \geq\left(\frac{a b C_{0}}{2(p+3)\left\|u_{0}\right\|_{L^{1}(\mathbb{R})} V^{2}\left(x\left(\alpha_{0}, t_{0}\right), t_{0}\right)}\right)^{1 /(p+3)}
$$

which gives

$$
\int_{t_{0}}^{G(t)} \frac{d \xi}{\sqrt{\xi^{p+3}+1}} \geq\left(\frac{a b C_{0}}{2(p+3)\left\|u_{0}\right\|_{L^{1}(\mathbb{R})} V^{2}\left(x\left(\alpha_{0}, t_{0}\right), t_{0}\right)}\right)^{1 /(p+3)}\left(t-t_{0}\right) .
$$

Taking $T$ to be

$$
+\infty>\int_{t_{0}}^{\infty} \frac{d \xi}{\sqrt{\xi^{p+3}+1}}=\left(\frac{a b C_{0}}{2(p+3)\left\|u_{0}\right\|_{L^{1}(\mathbb{R})} V^{2}\left(x\left(\alpha_{0}, t_{0}\right), t_{0}\right)}\right)^{1 /(p+3)}\left(T-t_{0}\right),
$$

when $t \longrightarrow T<+\infty$ in (53), we have

$$
+\infty>\int_{t_{0}}^{\infty} \frac{d \xi}{\sqrt{\xi^{p+3}+1}} \geq\left(\frac{a b C_{0}}{2(p+3)\left\|u_{0}\right\|_{L^{1}(\mathbb{R})} V^{2}\left(x\left(\alpha_{0}, t_{0}\right), t_{0}\right)}\right)^{1 /(p+3)}\left(T-t_{0}\right),
$$

which implies that the maximal existence time to the solution of (1) cannot be extended more than $T<\infty$. This is a contradiction with the assumption that the solution is global in time. The proof of Theorem 2 is complete.

\section{Data Availability}

No data were used to support this study.

\section{Conflicts of Interest}

The authors declare that they have no conflicts of interest.

\section{Acknowledgments}

The research of L. R. Li was supported by the Scientific Research Plan Projects for Higher Schools in Hebei Province 
(Grant no. QN2019308) and Special Fund of Fundamental Scientific Research Business Expense for Higher School of Central Government (Projects for young teachers, Grant no. ZY20180212). The research of S. Wang was partially supported by the National Science Foundation of China (Grant nos. 11531010, 11831003, and 11771031) and Qinghai Science Foundation of China. The research was also funded by the China Scholarship Council (no. 201902025001).

\section{References}

[1] P. Constantin, P. D. Lax, and A. Majda, "A simple one-dimensional model for the three-dimensional vorticity equation," Communications on Pure and Applied Mathematics, vol. 38, no. 6, pp. 715-724, 1985.

[2] J. M. Burgers, "A mathematical model illustrating the theory of turbulence,"Advances in Applied Mechanics, vol. 171-199, Academic Press, New York, NY, USA, 1948.

[3] E. Hopf, "The partial differential equation ut+uux $=\mu \mathrm{xx}$," Communications on Pure and Applied Mathematics, vol. 3, no. 3, pp. 201-230, 1950.

[4] S. D. Gregorio, "On a one-dimensional model for the threedimensional vorticity equation," Journal of Statistical Physics, vol. 59, no. 5-6, pp. 1251-1263, 1990.

[5] S. D. Gregorio, "A partial differential equation arising in a 1D model for the 3D vorticity," Mathematical Methods in the Applied Sciences, vol. 19, pp. 1233-1255, 1996.

[6] Z. Lei, J. Liu, and X. Ren, "On the Constantin-Lax-Majda model with convection," Communications in Mathematical Physics, vol. 375, no. 1, pp. 765-783, 2020.

[7] H. Okamoto, T. Sakajo, and M. Wunsch, "On a generalization of the Constantin-Lax-Majda equation," Nonlinearity, vol. 21, no. 10, pp. 2447-2461, 2008.

[8] A. Catro and D. Córdoba, "Infinite energy solutions of the surface quasi-geostrophic equation," Advances in Mathematics, vol. 225, pp. 1820-1829, 2010.

[9] A. Córdoba, D. Córdoba, and M. A. Fontelos, "Integral inequalities for the Hilbert transform applied to a nonlocal transport equation," Journal de Mathématiques Pures et Appliquées, vol. 86, no. 6, pp. 529-540, 2006.

[10] A. Córdoba, D. Córdoba, and M. A. Fontelos, "Formation of singularities for a transport equation with nonlocal velocity," Annals of Mathematics, vol. 162, no. 3, pp. 1377-1389, 2005.

[11] T. Sakajo, "Blow-up solutions of the Constantin-Lax-Majda equation with a generalized viscosity term," Journal of Mathematical Sciences Tokyo, vol. 10, pp. 187-207, 2003.

[12] T. Y. Hou, C. Li, Z. Shi, S. Wang, and X. Yu, "On singularity formation of a nonlinear nonlocal system," Archive for Rational Mechanics and Analysis, vol. 199, no. 1, pp. 117-144, 2011.

[13] K. Choi, T. Y. Hou, A. Kiselev, G. Luo, V. Sverak, and Y. Yao, "On the finite-time blowup of a one-dimensional model for the three-dimensional axisymmetric euler equations," Communications on Pure and Applied Mathematics, vol. 70, no. 11, pp. 2218-2243, 2017.

[14] A. Kiselev, F. Nazarov, and R. Shterenberg, "Blow up and regularity for fractal Burgers equation," Dynamics of Partial Differential Equations, vol. 5, no. 3, pp. 211-240, 2008.

[15] A. Castro, D. Córdoba, and F. Gancedo, "Singularity formations for a surface wave model," Nonlinearity, vol. 23, no. 11, pp. 2835-2847, 2010.
[16] V. M. Hur, "On the formation of singularities for surface water waves," Communications on Pure and Applied Analysis, vol. 11, no. 4, pp. 1465-1474, 2012.

[17] J. K. Hunter and M. Ifrim, "Enhanced life span of smooth solutions of a Burgers-Hilbert equation," SIAM Journal on Mathematical Analysis, vol. 44, no. 3, pp. 2039-2052, 2011.

[18] G. M. Coclite, S. Dipierro, F. Maddalena, and E. Valdinoci, "Singularity formation in fractional Burgers equations," Journal of Nonlinear Science, vol. 30, no. 4, pp. 1285-1305, 2020.

[19] X. H. Wang and J. H. Zhang, "Non-existence of positive solutions to nonlocal Lane-Emden equations," Journal of Mathematical Analysis and Applications, vol. 488, no. 1, Article ID 124067, 2020. 